\title{
Research on large section and broken coal roadway in Zhaozhuang Coal
}

\author{
Mine \\ Hanjun Wang ${ }^{1, \text { a }}$ \\ ${ }^{1}$ Beijing Polytechnic College, Beijing, 100042, China \\ awhjg8351@sina.com
}

\begin{abstract}
Key words: Large section broken coal roadway; large deformation; FLAC3D numerical simulation software; optimization support

Abstract. The Third mining area 33073 roadway of Zhaozhuang Coal Mine is a large section and broken coal roadway, after tunnel excavation, the emergence of large deformation surrounding rock phenomenon. By using simulation software roadway FLAC3D original support scheme simulation analysis, we found the original support scheme to control deformation of the roof and floor have a good effect, but the effect is poor with ribs of the roadway, then put forward three kinds of optimization support program, and its roadway displacement cloud for analysis. The results show that the Option 1 and Option 2 cannot support effectively to put roadway into good control. Support Schemes 3 through the tunnel roof support mode from full length anchor support to anchor and anchor combined support, to help reduce inter-row spacing of Bolt, change to help the Department of the top corner angle and anchor help section anchor arrangement allows the deformation of surrounding rock have been a more effective control, not only reduces the cost of support, but also reduces the time needed to support and improve the efficiency of support.
\end{abstract}

\section{Introduction}

Our country has large reserves of coal resources, buried in shallow, easy exploitation, etc., in China's energy consumption structure and long-term in a dominant position for the economic and social development provides a powerful energy force, to protect the security and stability of China's energy [1]. With the continuous damping shallow strata of coal resources, so increasing the depth of coal mining, mining and support increasingly complex conditions encountered [2]. Many of our mines have emerged in varying degrees of soft rock roadway using traditional methods are difficult to control, not only severely restricted the underground roadway driving speed, but also to pave the roadway excavation significant safety risks, an impact on our production and construction of major coal mine one of the technical problems [3].

Zhaozhuang Coal Mine in Shanxi as large mine, the design production capacity of over 6 million tons / year, cross-sectional area of the underground tunnel of generally more than $20 \mathrm{~m} 2$, is typical of large section roadway. Zhaozhuang ore region has a lot of geological structure and large water content, and high coal seam gas content, coal quality and top floor surrounding rock of the 3rd is soft, typical of the "three soft seam." Coal measures complex roof shale contains instability thickness, poor roadway conditions, empty-support distance is small, so large section roadway mechanized tunneling is difficult to fully play its advantages, roadway driving speed affected is very large, the monthly footage often less than $300 \mathrm{~m}$, and roadways and chambers have suffered different degrees of deformation, some regions even suffered different degrees of de-anchor phenomenon, which greatly affect the roadway excavation rapidly, mines continue mining and 
production safety. This paper intends to combine large section Zhaozhuang ore mining area with complex roof site engineering conditions, using numerical simulation method to Zhao Mine Coal Lane 33073 Third District for the study and grasp the laws of deformation and failure of surrounding rock, supporting identify parameters for the coal Lane, to reduce the cost of supporting and repairing the roadway, underground production to ensure security purposes.

\section{Project Overview}

Zhaozhuang Coal Mine Third District 33073 is intaking air, haulling, intaking shelf roadway, the ribs is 3 \# coal, rectangular roadway, along the top and preserve the bottom driving, roadway width $5000 \mathrm{~mm}$, height $4500 \mathrm{~mm}$, driving cross section area of $22.5 \mathrm{~m}^{2}$, is a large section Roadway. The average thickness of $3 \#$ coal seam is $4.7 \mathrm{~m}$, complex structure, mainly as clarain, showing irregular-shaped fracture, massive portions contain partings, lithology is mudstone partings, and contains durain section. Seam dip between $3 \sim 5 \circ$ ․ 33073 roadway design to uses anchor cable combined supporting. Roof anchor chosen diameter $22 \mathrm{~mm}$, length $6.4 \mathrm{~m}$ high strength prestressed steel strand anchor, row spacing $1200 \mathrm{~mm}$, distance $1100 \mathrm{~mm}$, roadway anchor and angle at a distance with the help of the Ministry of $300 \mathrm{~mm}$, each row of five anchor design and installation; Lane help anchor the choice of diameter $22 \mathrm{~mm}$, length $2.4 \mathrm{~m}$ steel bolt, the row spacing $1200 \mathrm{~mm}$, spacing $950 \mathrm{~mm}$; roadway anchor chosen diameter $22 \mathrm{~mm}$, length $5.4 \mathrm{~m}$ high strength prestressed steel strand anchor, row spacing $2400 \mathrm{~mm}$ pitch $2200 \mathrm{~mm}$. Because Zhaozhuang Coal Mine mining area relatively soft and prone to breaking, and confining pressure greater depth roadway caused also larger, resulting in a roadway deformation is relatively large, it is necessary to optimize the existing forms of support.

\section{Original model}

FLAC3D numerical simulation model to determine the scope of a direct impact on the accuracy of the results, due to the stress generated after tunnel excavation roadway will have different degrees of impact, stress seen by the rock mechanics radius excavation roadway width of $3 \sim 5$-fold. Zhaozhuang Coal Mine Third District 33073 roadway excavation width $5000 \mathrm{~mm}$, height $4500 \mathrm{~mm}$, so the size of the length and breadth of the entire model is set to $45 \mathrm{~m} \times 6 \mathrm{~m} \times 45 \mathrm{~m}$. In order to improve the accuracy and speed of calculation, grid around the roadway is more dense, other locales are relatively sparse, the model is divided into 32,382 units, 38,577 nodes. From top to bottom the entire model are sandy mudstone, medium-grained sandstone, sandy shale, coal, medium-grained sandstone, sandy shale and mudstone. After the model is applied to the initial stress, the stress of roadway from all directions: $\mathrm{Sxx}=16.7 \mathrm{Mpa}, \mathrm{Syy}=16.8 \mathrm{MPa}, \mathrm{Szz}=16.0 \mathrm{MPa}$, based on stress test results, in line with the initial stress of roadway actual situation. Establishment of the original model is shown in Fig. 1. Numerical analysis uses mole Coulomb constitutive

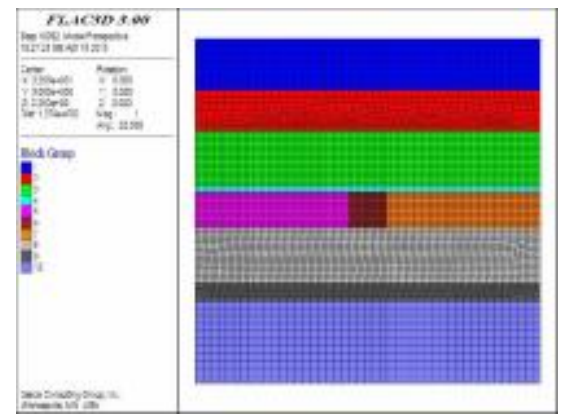

Fig. 1 Diagram of the original model

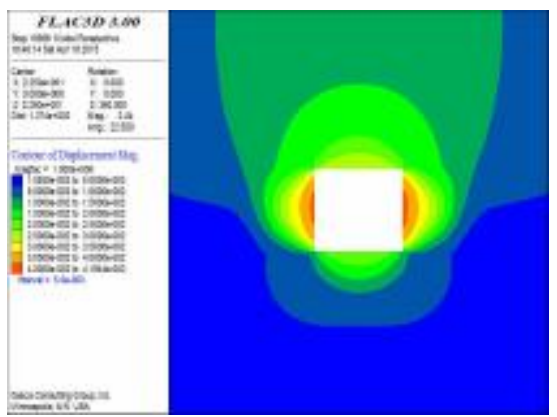

Fig.2 Displacement image 


\section{Simulation of the original support scheme}

Model calculation to plastic equilibrium state, the model displacement is resetted, according to the actual conditions on the 33073 section of roadway a full excavation, then according to the above arrangement of the original support scheme to Bolt (Cable), and to count to calculate balance, and ultimately to obtain the results of the original support under the program. According to the research focus of this article, collect and analyze the results.

Displacement image in original supporting state is shown in Fig. 2. Fig. 2 shows that the deformation occurs mainly in the roadway two sides roadway, and gathered in the middle of the roadway about two to help, displacement has reached $41.8 \mathrm{~mm}$. Top floor displacement is relatively small compared to the two to help, the roof is about $27.5 \mathrm{~mm}$, the floor is about $23 \mathrm{~mm}$, described the original support scheme does not produce effective control with the help of the Department of rock deformation, while the top and bottom of the deformation from to a good result.

\section{Optimizing Simulation of support programs}

Option One. Roof anchor support from the five to two, the middle and both sides of the anchor bolt is replaced, to 2 anchor with 3 anchor cable combination support, design row spacing of $1200 \mathrm{~mm}$, $1100 \mathrm{~mm}$ pitch. With the help of the rib support bolt row spacing $1200 \mathrm{~mm}$, spacing reduced to $900 \mathrm{~mm}$, playing an angle bolt, bolt from the bottom corner $500 \mathrm{~mm}$. Top corner anchor and $10^{\circ}$ to the horizontal angle $30^{\circ}$, the other two are related to the roadway wall vertical. Each anchor is arranged to help two per row, row spacing is $2400 \mathrm{~mm}$, the upper part of a roof anchor from $800 \mathrm{~mm}$, anchor spacing $2200 \mathrm{~mm}$, the lower part of one anchor from the floor $1500 \mathrm{~mm}$.

Option Two. Roof anchor support from the five to three, in the middle and both sides of the anchor unchanged, replace the two remaining anchor, to 2 anchor with 3 anchor cable combination support, design row spacing of $1200 \mathrm{~mm}$, spacing of $1100 \mathrm{~mm}$. With the help of the rib support bolt row spacing $1200 \mathrm{~mm}$, distance $1200 \mathrm{~mm}, 400 \mathrm{~mm}$ corner bolt from the floor. Bolt top corner angle and horizontal angle to keep the original program unchanged, others are related to roadway vertical. Anchor arrangement becomes 2-1-2 streaky arranged every two to help each row of each anchor, row spacing is $2400 \mathrm{~mm}$, the upper part of an anchor from the roof $800 \mathrm{~mm}$, anchor spacing $2200 \mathrm{~mm}$, from the lower part of an anchor floor $1500 \mathrm{~mm}$, the middle one is located with the help of the central portion of the anchor.

Option Three. Supporting anchor the top five three, the middle on both sides and in the middle unchanged, and the remaining two bolt replaced, instead combination support three anchor cable with 2 anchor, row spacing reduced to $1100 \mathrm{~mm}$, spacing to $1100 \mathrm{~mm}$. With the help of the rib support bolt row spacing $1100 \mathrm{~mm}$, spacing reduced to $900 \mathrm{~mm}, 500 \mathrm{~mm}$ corner bolt from the floor. Angle bolt directly hit the top corner, anchor and horizontal angle of $30^{\circ}$, corner anchor and horizontal angle of $30^{\circ}$, the other are the roadway perpendicular. Anchor arrangement becomes 2-1-2 streaky arranged two each to help each row, then row spacing is $2200 \mathrm{~mm}$, the upper part of a roof anchor from $800 \mathrm{~mm}$, anchor spacing $2200 \mathrm{~mm}, 1500 \mathrm{~mm}$ plate from the lower part of one anchor the middle portion of a central help anchor portion.

Displacement images in three supporting schemes are shown in Fig. 4. Fig. 4 shows that the maximum displacement occurred in the roadway of the two groups, the overall displacement of the three supporting programs to help the two were: $55.5 \mathrm{~mm}, 51 \mathrm{~mm}, 42.2 \mathrm{~mm}$, the overall ceiling of the amount of displacement is relatively small, and three support top floor overall amount of displacement of less state, respectively: $37.5 \mathrm{~mm}, 32.4 \mathrm{~mm}, 37.5 \mathrm{~mm}$, described the three support programs in the inhibition of the roof and the bottom floor heave roof Fall aspects can play a good 
effect. Supporting Scheme 3 only for the roof and floor displacements with very good results overall, but also effectively suppress the deformation of the two help to ensure that the overall shape of the roadway section.

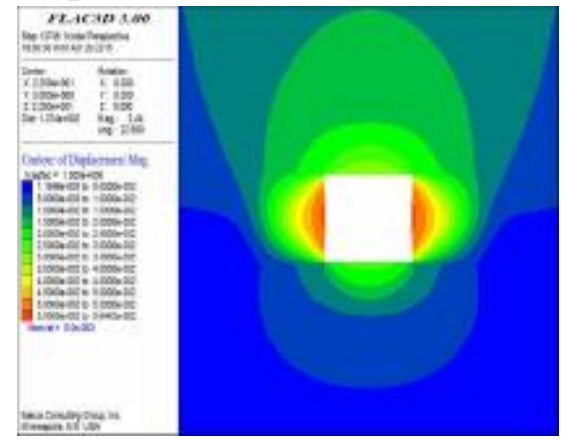

a Support plan 1

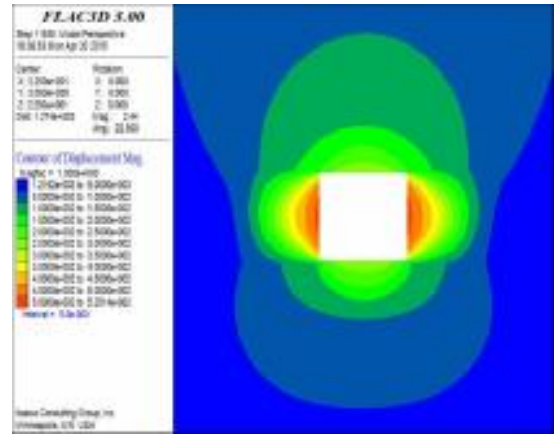

b Support plan 2

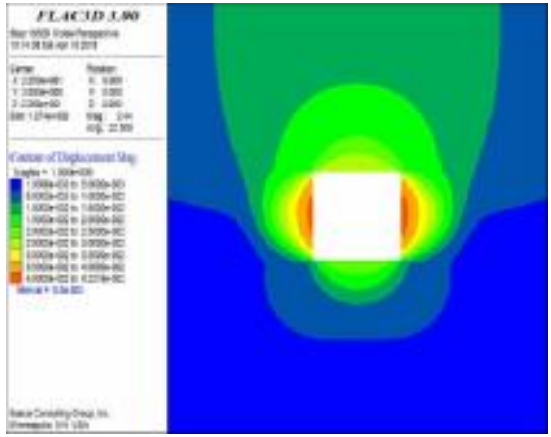

c Support plan 3

Fig.3 Displacement images in different supporting schemes

\section{Conclusion}

The use of numerical simulation software FLAC3D in Zhaozhuang Coal Mine Third District 33073 Large Section Coal Roadway Broken original support scheme simulation analysis, the original support scheme to control deformation of the roof and floor with good results, but the side control results is poor.

According to the analysis, Zhao Mine Third District 33073 Large Section Coal Roadway broken proposed three different optimization support program, and three kinds of roadway displacement of cloud optimization support under the program, support Schemes 1 and 2 are not supporting programs to help effectively deformed roadway good control. Support Schemes 3 through the tunnel roof support mode from full length anchor support to anchor and anchor combined support, to help reduce inter-row spacing of Bolt, change to help the Department of the top corner angle and anchor help section anchor arrangement allows the deformation of surrounding rock have been a more effective control. Supporting Option 3 takes full advantage of the strength of the tunnel roof itself, reducing the number of top anchor not only reduces the cost of support, but also reduces the time needed to support and improve the efficiency of support.

\section{Acknowledgements}

This work is financially supported by the Project of Science and Technology Innovation Platform of Beijing Municipal Education Commission of China (PXM2013_014225_000051) and Science and Technology Development Project in 2015 of Beijing Municipal Education Commission of China (KM201510853002).

\section{Reference}

[1] Yanhong Wang, Meng Wang. Prospect Research on deep mine mining problem and development [J].Coal Technology, 2008, 27(1): 86-87.

[2] Jinhua Wang. The new development of coal roadway bolting technology in China [J]. Journal of Coal, 2007, 32(2): 113-118.

[3] Feng Yang. Deformation and failure characteristics of high stress soft rock tunnel excavation and crush supporting mechanism research [D]. Xuzhou, Jiangsu: China University of Mining and Technology, 2009. 